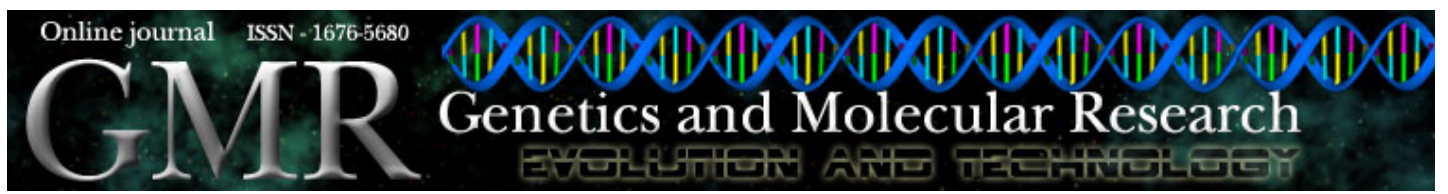

Point of View

\title{
The future of selection: individuality, the twin legacies of Lamarck \& Darwin
}

\author{
Hugo Hoenigsberg \\ Departamento de Genética \& Evolución \\ Universidad Manuela Beltrán \\ Bogotá, D.C. \\ Colombia \\ E-mail: hoenisbe@www.umb.edu.co
}

Key words: Future selection, Lamarck \& Darwin legacies, Evolution, Immune system, Somatic selection

Genet. Mol. Res. 1 (1): 39-50 (2002)

Received February 8, 2002

Published March 6, 2002

\section{INTRODUCTION} selection:

I discuss two modern developments in evolution that will take part in future studies of

1) The different levels of biological hierarchy - genes, chromosomes, cells, species and communities - are different survival entities that require different heritable variations in fitness in order to function as units of selection in the evolutionary process. These entities have retained to varying degrees some of their primordial lower-level identity, where selfish survival of genes was instrumental to better and multiply their type. That lower-level unit was natural selection's milieu. The evolutionary process went through transitions in the units of selection, from protogenes to gene networks and to the various biological steps in the hierarchy of life by inventing cooperation among the lower-level units for the functioning of the higher-level unit. We argue that the invention of cooperation among networks of genes was possible through regulation of the primordial paradigmatic conflictual and selfish program. Eigen and Schuster (1979) proposed the hypercycle as a way to keep individual genes from competing among each other so that cooperating gene groups could emerge. Chromosomes reduce the conflict among individual genes (Maynard Smith and Szathmáry, 1995). Meiosis serves to police the selfish tendencies that prevail in the genome from early programs. Diploidy was an early invention to suppress the deleterious consequences of selfishness. Uniparental inheritance of 
cytoplasm reduces conflict among organelles through the expression of nuclear or organelle genes (Hoekstra, 1990; Godelle and Reboud, 1995). Finally, other units, like kin selection, reciprocation, frequent encounters and group structure regulate conflict in the final transitions from organisms to societies of cooperating organisms.

2) Weismann's legacy is that the soma and the germline are irremediably separated and therefore genetic variation arising during the course of ontogeny cannot be inherited. In Weismann's theory the implication is that the individual is a unique genetically homogeneous entity. In future studies of selection evolutionary geneticists will have to recognize that their past preformistic modes of development (Drosophila, Aves, Mammals) were biased in their generalizations. Our Professors, Dobzhansky, Mayr, Simpson, Timofeef-Ressovsky, BuzzatiTraverso and Rensch were experts in Dipterans and vertebrates. We forgot that there are other modes of development: somatic embryogenesis, epigenesis and preformation. If we take all these modes into consideration we conclude that heritability is controlled by development, as Weismann recognized. Unicellular organisms can reproduce clones of themselves with just mitosis, in which all descendants are capable of giving rise to a new multicellular individual, while only a fraction of the cells of metazoan zygotes dividing by mitosis can produce individuals. This is an important difference, as the former all cells retain totipotency and succeed in asexually producing a new organism capable of genetic immortality. On the other hand, only by sequestering a specific cell lineage can preformistic metazoan development reach gamete formation and totipotency ....and immortaliy (for a more critical review of this subject see Buss, 1987).

In somatic embryogenesis a distinct germline is lacking. Moreover, any cell lineage is capable of both somatic functions as in a stem cell lineage and reproduction through gamete formation (e.g., plants). In contrast organisms with epigenetic development (e.g., annelids, nemertina, echinodermata) possess a clearly differentiated germline although it appears late after the primordia of tissues and organs of the adult have developed fully. The preformistic mode of development has the germline terminally differentiated in earliest ontogeny, frequently maternally directed through molecular signals deposited in the egg (e.g., nematodes).

The phyletic distribution of developmental mode is fairly clear. Somatic embryogenesis is by far the most common mode of development. Only representatives of the Kingdom Animalia possess epigenetic and preformistic development. At least nine animal phyla display somatic embryogenesis throughout their life cycle. This fact means that it is inaccurate to consider the individual as the only unit of inheritance in most taxa.

We hope that future studies of selection take into consideration the fact that plants, colonial invertebrates and fungi all violate Weismann's doctrine, displaying enormous phenotypic plasticity. As we will see later on in this paper different levels of selection are possible in the Animal Kingdom. Moreover different strategies have developed in the evolution of transitions between the unicellular and the multicellular, and these have emerged according to the presence of individuality in the various taxa. It is of outmost importance to recognize that the Modern Synthesis has not generated a terminal theory of ontogeny.

3) The latter part of this essay shows how Lamarckian inheritance at the molecular genetic level fits in.

\title{
WHERE DO ORGANISMS COME FROM? THE SOLUTION OF CONFLICTS BETWEEN CELLS
}

\author{
Michod's multilevel selection framework (Michod, 1997a,b, 1999) is an attempt to
}


answer the dominant feeling in Neo-Darwinian thinking that organisms are the sole units of selection (Morrell, 1996). Paleontological evidence (Xiao et al., 1998) of the developmental forms of ancestors of metazoans suggests that the entire ontogeny of the ancestors of multicellular animals was probably similar to the type 1 embryogenesis of modern groups (Davidson et al., 1995).

The different steps in the biological hierarchy - genes, chromosomes, cells, organisms, kin groups, societies and communities - undoubtedly have varying degrees of coordination and cooperation of the different steps that require different expressions of heritable differences in fitness. This is the same as saying that each step (or some of them) may function as unit of selection in the evolutionary process in different ways. No wonder the study of transition from unicellular to multicellular organisms has focused on understanding transitions between different levels of selection (Maynard Smith and Szathmáry, 1995).

The major transitions in evolutionary units that are relatively easy to identify are individual genes or protogenes to networks of genes, from the latter to Hydra-like unicellular organisms, from Hydra-like cells to complex eukaryotic cells with organelles, and finally to multicellular organisms, species and societies. These transitions in the ways in which the units of selection operate, have in common their commitment to cooperation among the lower level units to jump to the new higher-level unit, and the control of the conflicting genomes among the lower-level units where natural selection acts undisturbed. The various strategies in which the units of selection operate to jump to the new higher level unit depend on the evolutionary course of development: in cells like Hydroids heritability is controlled by development. While in Drosophila the totipotent lineage undergoes only 13 nuclear divisions per sexual generation, in Hydra the same lineage may undergo an astronomical number of divisions before sexuality appears (Buss, 1987). The potential for variation in Diptera is very low while in Hydra it is enormous. The asexual reproductive phase of Hydra may be of indeterminate length.

The transition from unicellularity to higher levels of multicellularity set in much before the phyletic distribution of development arose. This early transition before cellular differentiation and the developmental mode responded to local environmental insults deserves a theoretical treatment.

Eigen and Schuster (1979) proposed the hypercycle as a means to keep individual genes from competing among themselves so that cooperating gene networks could emerge. The ability to identify genes in the cell keeps selfish parasitic genes from destroying the cooperative nature of the evolving genome. Chromosomes could be one of the first units to reduce conflict among the individual genes (Maynard Smith and Szathmáry, 1995). Meiosis serves to police selfishness of genes and uniparental inheritance may serve as a means of reducing conflict among organelles by limiting the expression of either nuclear or cytoplasmic genes (Hoekstra, 1990; Godelle and Reboud, 1995). Moreover, kin selection or group selection, repeated encounters and group structure may serve to regulate conflict, or at any rate to control the selfish tendencies of the lower-level units for the necessary cooperation of genes to evolve to the higher-level unit of multicellular organisms.

Some have proposed that control of cell behavior is paramount to reduce conflict among maternal cells (Buss, 1987). Similarly, Michod (1982) indicates parental control rather than offspring control in the evolution of altruism. Michod (1999) argues that "by limiting development to a small number of cell divisions under the control of maternal cytoplasm, along with strictly determined cell fate prior to the movement of any cells, the opportunity for conflict should be reduced".

It is conceivable that the ontogenetic characteristics that evolved from the ancestors of multicellular life forms are the result of maternal control and kin selection. This evolution 
reduced conflict among cells and promoted harmonious behavior within the group even at the expense of not maximizing fitness and adaptation. According to Davidson et al. (1995), in their developmental regulatory mechanism, the undifferentiated "set-aside" (stem cells) cells are the key to more complex structures because of the unlimited replication of these cells. Following the logic of Davidson, defecting mutation of undifferentiated stem cells would increase risks for disorders in the organism because conflict without modifiers would increase, and pathology, and maybe death, would ensue (Ransick et al., 1996).

For a new level of selection to start it is important for primitive unicellular life to have solutions to conflict, and the genetic mechanism for their regulation firmly established even before cell divisions initiate their movement toward other cells. Michod (1999) argues that early conflict regulation can be accomplished by reducing generation time, $t$, and by maternal control, and that both can be prevented by stem cells with unlimited replication potential. Thus, a large population of set-aside cells can evade conflict modifiers. In order to mediate conflict and proceed with the evolution of more ontogenic processes in such a large population of cells, germline modifiers become necessary. The unit of selection at this point of development is still the allele that confers better conflict control through the evolution of the germline and other self-policing modifiers at that early level.

However, the unit of selection for the adaptation of modern organisms depends on a step by step developmental process. In the sea urchin, where the cell types in the adult are determined after embryogenesis and development of the larva, the unit of selection of the second process for building the adult form is on another level (the ancestral type 1 mode of development). Since cellular conflict can come into play only after type 1 development is complete, then germline sequestration can be involved and be of use only when the first rigidcontrol-conflict has been organized in toto. Which, by logical deduction, means that the step by step development of the modern organism has at least two levels of selection acting in cooperation and in coordination and that the overall adaptation is the result of the new coherent unit that ends up at a new level of organization. In other life forms such as in arthropods, nematodes and chordates there is direct development, that is, cell types are specified during early cleavage of the embryo and therefore primitive type 1 development has been avoided with the concomitant result that primordial specification of the germline occurs at gastrulation because defecting mutants may arise quite early and threaten the integrity of the organism. In this kind of direct development, the organism, at another higher level of selection, had to have the previous unit well organized at the intercellular population level in order to reduce the opportunity for the emergence of within-organism conflicting genomes.

\section{THE CHALLENGE OF THE CONCEPT OF FITNESS}

The challenge to the modern evolutionist is how to conceptualize fitness in the realm of transmission and heritability so that the units of selection, as they are involved in a choice, do not turn out to be a poor Neo-Darwinian description of the actual dynamics involved in the transition from single cells to multicellular organisms. The hypothesis that the germline and the self-policing system evolved a progressive adaptation of reducing and controlling withinorganism change, because this orthoselective mechanism presumably served to facilitate the transition between cells and multicellularity, should be seriously considered. Genetic homogeneity reduces selection. Early germline sequestration would reduce the opportunity for conflicting genomes and at the same time would get out of selection's reach because cells would tend to be homogeneously cooperative and cell duplication would be limited or would 
produce similar cells. Moreover, intracellular or intercellular mechanisms tending to reduce within organism change will be favored not by selection but by the intercellular mechanisms that promote fusion through cohesion and cooperation as in Myxobacteria and slime molds. In fact this new mechanism may be considered an organismic-multicellular-organization, nonDarwinian, but only while homogenous cooperation lasts or exists to promote a higher level system (e.g., a tissue, organ, etc.) necessary for higher-level-system survival. This latter is a more inclusive evolutionary unit. Clearly, "jumping" to cooperation through conflicting genome modifiers of various types means reducing the fitness of lower-level units but at the same time increasing the fitness of the multicellular "group". There may be moments between unicellular cooperations and the new higher-level-system in which multicellular organization has not yet set in, in which case the evolutionary transition is left without selection of the lower level or without the higher more inclusive evolutionary unit. There, we will have "blanks" and no evolution. How long these "uncertain" periods will last, no one can predict, simply because they will depend on nature's circumstances. Since desertion from cooperative arrangements may result from mutation among conflict-gene-modifiers, the transition has to wait for the mechanism of cooperation to initiate the reduction of fitness of lower level units. Mutation rate and the frequency-dependent advantages that result within the population of cells have to be at a minimum and reduced to just some cells, but not all. Frequency-dependent selection within the population will always boycott the maximization of group fitness. The transition period cannot be bountiful in its new fitness function with those mutation-based frequency-dependent selection individuals. Their rapid elimination will make way for the higher level unit. Adaptation as viewed by traditional Neo-Darwinism (although not by Darwin) (e.g., Williams, 1996) is set to produce only better-designed individuals (the phenotype) sacrificing the other levels of organization where conflict mediation made life so bountiful in many directions.

\section{THE TWIN LEGACIES OF LAMARCK AND DARWIN}

The past 45 years have seen an explosion in our knowledge of basic molecular genetics and this recent revolution is transforming our view of the mechanisms of inheritance and the evolution of life on Earth. The time is now ripe to see and analyze the implications to the basic tenets of evolutionary theory. The scientific revolution initiated by Charles Darwin, that natural selection is the driving force in evolution, has become stagnated with Neo-Darwinian thinking. We believe the dogma requires updating with the new perspectives of the molecular revolution in the immune system of animals with backbones, the vertebrates. Therefore, it is now reasonable to entertain previously heretical concepts and questions such as: Is there a Lamarckian principle at work in the immune system? Is Weismann's Barrier permeable to some extent? In other words, can some acquired characteristics be inherited? Can we describe the process in molecular terms?

The key conceptual point is this: mutations in genes of somatic cells of an animal can possibly be transmitted to the genes of germ cells and passed on to offspring of future generations. This is a molecular update to the original idea of the inheritance of acquired characteristics presented by Lamarck and later accepted by Darwin in his Pangenesis theory.

We therefore introduce the two, not necessarily incompatible, concepts: 1) the traditional Neo-Darwinian that evolutionary genetic variability pre-exists before the selective force acts (natural selection); versus, 2) the Lamarckian view of the generation of genetic variability at the same time as the selective force acts. This latter concept is singularly relevant to the immune system, where the selective force/environmental stimulus, the infectious disease, exists at the 
same time as the appearance of new somatic genetic variability (the mutated genes encoding antibodies against infection).

\section{Extra-Nucleic L Systems}

Just as it is for the immune system, the modern Lamarckian molecular process might explain why some species have been able to undergo an apparent rapid genetic transformation when sudden environmental changes, or catastrophes have occurred in nature.

Cluster analysis carried out with 16 ecotypes of Hydra collected from 15 widely separated and ecologically distinct localities in India has revealed that local adaptation is simply the result of switching specific genes, depending on the ecological requirements (Rastogy and Pandey, 1992). This unicellular animal has a tremendous regulatory machinery of structural reorganization that constitutes a challenge to traditional taxonomy (Ewer, 1948; Grayson, 1971; Campbell, 1983). As with protozoa, Hydra resort to sexual reproduction only under unfavorable environmental conditions (Prasad and Mookerjee, 1986). Hydra's universal adaptation forces the experimenter to adopt an L system model in view of the fact that gene frequency changes are not a prerequisite to adaptation. A single individual can do it with an extranuclear trigger superadaptability that probably retained the ancestral form of modern marine larvae that have "type 1" development, which is a widespread and basic mode of embryogenesis in modern animals.

In type 1 development cleavage begins immediately after fertilization and proceeds for a number of cell divisions, as in sea urchin embryos. By the end of cleavage, all the blastomeres have been specified; the resulting embryo has one thousand cells and is divided into a group of polyclonal lineages in which each element gives rise to a certain differentiated cell type. Following Blackstone and Ellison (1998) one can interpret type 1 development as a means of reducing the time for development and the intercellular variability through defecting cells, by way of maternal control. Maternal control of cell behavior (first proposed by Buss, 1987) in the transition of unicellular Hydra-like animals to multicellular ones retaining L type extranucleic inheritance can be a way of reducing conflict among cells, thereby making cooperation possible.

Thus, Hydra's L type superadaptability could be the origin of multicellular life that went through an evolutionary transition to a new higher-level unit of individuality.

There have been cases in Drosophila in which the comparative genetic structure and spatial patterns of cosmopolitan and local species have shown rapid genetic changes among profoundly isolated local demes that cannot be interpreted with the usual Neo-Darwinian theory (Hoenigsberg and Parkash, 1995). Jenning's (1940, p. 48) clearly stated that: "The doctrine of the inheritance of acquired characteristics finds its last refuge in the genetics of Protozoa. It is a fact that Protozoa are modified in many ways by the action of environmental conditions, and it is known that the modified characteristics so induced are inherited for long periods in vegetative reproduction for hundreds of generations" (quoted from Sapp, 1987). Moreover, some authors have analyzed epi- and extranucleic inheritance, and have found it necessary to postulate Lamarckian inheritance and non-Mendelian inheritance (Landman, 1991; Hoenigsberg, 1992).

\section{The Somatic Selection Hypothesis}

The Somatic Selection Hypothesis, first proposed by Steele (1979), is a modern molecu- 
lar counterpart of the Pangenesis idea. This hypothesis proposes a mechanism to explain the genetic evolution of variable antibody genes (V genes) via a soma-to-germline gene feedback system. The mechanisms allow for the creation of new genetic variant animals in response to microbial invaders from the external environment. There is now no doubt that the DNA sequences which encode the proteins for the recognition of foreign invaders (antibodies) undergo rapid somatic gene mutation as a result of being activated by the antigens of the invading infectious agent. The most recent data strongly support the theory that antibody gene mutations are passed back to germline DNA in a process involving reverse transcription. Reverse transcription, after a stormy reception, has been accepted after a Nobel Prize was awarded to Temin and Baltimore (1975) and it has now become widely recognized as an essential process in the replication of retroviruses (such as HIV) and other cellular events. Retroviruses are so-called because genetic information flows from RNA to DNA, the reverse of the normal direction, DNA to RNA, in all living cells.

For a general summary of the essential points concerning the immune system as a Lamarckian process in the Darwinian controversy we offer Rothenfluh and T. Steele (1993); Rothenfluh and E.J. Steele (1993); Steele et al. (1996, 1998). Most of the detailed molecular evidence can be found in Rothenfluh et al. (1995). To analyze Weismann's barrier we offer Pollard (1984) and Buss (1987).

The evidence points to the operation of the gene feedback loop involving the flow of genetic information from somatic cells (lymphocytes) into the germline genes.

The appearance in the germline of a gene structure (a specific gene sequence) thought to be present only in the soma cannot be ignored. Now that the human genome sequencing has been completed, we would not be surprised if the gene structure found in the soma is also found in the germline of all vertebrates. Lamarckian possibilities will have to be admitted as a general scientific interpretation in biology.

For the variable genes of the immune system one can say: How do we explain the presence of highly non-random patterns in the germline V gene DNA which can only arise through direct antigen-binding selection mechanism acting on the protein product of the gene (the antibody) instead of the DNA?

Steele's group at the University of Wollongong in Australia, ask the question of whether the DNA sequences of germline V gene benefit in any way from the antigen-driven somatic mutation and selection events that have occurred in previous generations. The question is: How many new V region mutant DNA sequences appear in B lymphocytes during immune responses and are subject to selection in view of the success of the encoded antibody in competing for antigen. Steele's group asks if these new sequences can pass over the germline DNA (Steele et al., 1998).

The Somatic Selection Theory predicts the germline transmission of acquired somatic mutations of antibody $\mathrm{V}$ region genes. This stunt could be effected through the enzyme reverse transcriptase (copying somatic RNA into DNA) mediated by naturally occurring endogenous RNA retroviruses (lymphocytic ones) acting as "gene shuttles" which transport mutated V region gene sequences into germ cells. The next step would be the physical integration of this somatically derived hereditary information into the germline DNA so as to replace the preexisting sequence.

It maybe interesting to remember that in the Proceedings of the National Academy of Sciences of the United States, Bartl et al. (1994) speculated that genes could be transmitted between species by viral infection, thus contributing to the evolution of the vertebrate immune system. Thus, last century, Weissman's barrier was already penetrated. This statement in the prestigious Proceedings is even more daring than Ted Steele's idea in the Somatic Selection Theory. 


\section{BACK TO DARWIN AND BEYOND DARWIN INTO LAMARCK}

Let us mention a few examples of acquired characteristics:

\section{Inheritance of Acquired Callousing (Wood Jones, 1943)}

Animals such as the ostrich and the African warthog have large callosities in parts of the body (sternum, forelimbs, hind limbs) as a consequence of their resting. The strong horny calluses appear to protect the skin surfaces upon which the animal kneels. Ostriches rest by squatting on their legs and their breast-bone (sternum). In these and in other animals, callusing can also be induced to occur if surfaces are subjected to frequent rubbing. Therefore, they can be classed as an "acquired" somatic adaptation. What is particularly interesting is that all those prominent natural calluses found in ostriches and warthogs are already well formed in the embryo in the absence of friction or rubbing. This means that these strategically located callosities are germline encoded. The skin pads of the soles of the feet of the newly born humans can be placed in this class.

Traditional Neo-Darwinian explanation (not by Charles Darwin but by his followers) is that these are peculiarities of a germline origin brought about by the natural selection process. Evidently to solve the issue between Neo-Darwinian explanation and the Lamarckian one requires experimentation with the genes involved. This is a very difficult and complex phenomenon, certainly more complex than the antibody V genes of B lymphocytes.

\section{Acquired Inheritance in Bacteria?}

Steele and Cairns (1989) and Howard Temin (1989) reviewed the evidence for the existence of reverse transcriptase enzymes in bacteria.

Without going into the details, John Cairns and co-workers of Harvard University were able to produce gene mutations in bacterial cultures in a directed fashion. They showed that certain mutations only appeared if chemical substrates related to the enzyme-encoding genes that mutated were present in the growth medium. Although controversial, John Cairn's original explanation made it necessary to invoke a reverse transcriptase-based gene feedback loop. Previously Steele (1979) used the same explanation for his soma to germline theory for the antibody $\mathrm{V}$ genes of the immune system.

\section{Acquired Inheritance in Plants?}

What about acquired inheritance in plants where there is no "Weismann Barrier" separating the soma and the germline. Acquired somatic modifications in plants which are the result of somatic mutations can be propagated to progeny when the seed is formed from that part of the plant that developed the somatic mutation. Therefore, Lamarckian evolution is and has been a fact in plants.

Phenomena such as induced heavy metal tolerance as acquired inheritance have been routinely demonstrated (McClintock, 1978; Cullis, 1984; Pren Das et al., 1990; Rothenfluh, 1995). 


\section{Acquired Inheritance in Mice?}

This example may not be a so very special scenario because it is known that mouse sperm cells maturing in the epididymal canals of the testis have the spontaneous ability to take up exogenous DNA which can be integrated into the genomic DNA of the sperm nucleus.

Spadafora and colleague's work (Zoragi and Spadafora, 1997) has been controversial but now they are defining many of the steps of the DNA-uptake pathway.

\section{THE IMMUNE SYSTEM}

Every day and every minute vertebrates are subjected to a relentless barrage of potentially invasive pathogenic viruses and bacteria. How does our immune system fight successfully such a multitude of pathogens?

Moreover, precisely because of its immense job, the immune system is far from being at harmony with itself. There is always the potential for a thorough full-scale "immunological warfare", in which even intracellular and within tissue immune responses react to fight off foreign invaders. Health means the ability to quickly make whole armies of new antibodies to fight previously unknown pathogens!

Thus, we should remember that we have a very intelligent system that enables pathogen recognition and regulation of immune responses, as well as generation and maintenance of immunological memory. The battle starts during late development of the embryo. The cells of the system first have to face their own molecular components ("self antigens"). Shortly after birth and environmental exposure, the immune system must then adapt to fight off foreign antigens. The fundamental problem for the system is being "designed" to fight a vast range of foreign pathogens, is how do they shun destroying "self". Another question is, since the molecular surface of the pathogens cannot be known in advance, how does an animal respond to the unexpected? And again, how does the system "remember" the molecular characteristics of previous pathogens so that they can be fought better at the second or subsequent encounters? And again how large is the repertoire of antibodies?

Landsteiner's amazing finding was that he could elicit antibodies against all of the new chemicals and drugs produced during the years. These new antigens never existed in nature! This means that there Neo Darwinian selective pressure could not exist for antibody production against a substance that did not exist!

The most mind boggling dilemma is that the potential repertoire of specific antibodies had to be enormous; and how can such a system evolve to make antibodies that were not part of the evolutionary history of the animal? If infectious diseases have anything to do with the evolutionary development of the modern immune system, then the molecular surface of the antigens have not been the driving force. It is more likely that a special biological strategy emerged to make an immune response to the unexpected.

Even in the cold blooded vertebrates, the fishes, the immune system of higher warmblooded vertebrates (birds, mammals including primates) can be found. All these immune systems have: 1) an enormous repertoire of antibodies and T cells which facilitate a response to virtually any antigen; 2) an enhanced (memory) response to future foreign infections, and 3) the possibility to maintain self-tolerance. 


\section{THE STRUCTURE OF ANTIBODIES}

The basic structure of antibodies was first described during the 1960's by a group led by Gerald Edelman and Rodney Porter, who shared a Nobel Prize in 1972.

Briefly, the antigen-binding site is composed of the complementary folding of the variable region $(\mathrm{V})$ provided by heavy $(\mathrm{H})$ and light $(\mathrm{L})$ protein chains called an HL heterodimer. Each antibody molecule has two identical HL heterodimers, except for pentameric IgM which has $10 \mathrm{HL}$ heterodimers. The constant (C) regions of the molecule trigger the lysis or phagocytosis of foreign bacterial cells and particles once the antibody has united its target antigens.

Conventional genetics says that one gene would be needed to encode an antibody $\mathrm{H}$ chain and that a second gene would be needed for the L chain. Is there sufficient length of DNA in our genome to encode millions of antibody specificities? This used to be a very important question in the sixties, as the Genetic Code was being deciphered. This question, plus the whole idea of self-tolerance, forced attention on to what type of strategy the immune system must employ to generate the diverse repertoire of antigen-binding receptors necessitated to fight off infectious diseases. Can the germline strategy, whereby all antibody specificities are encoded in the egg and sperm cells, be enough to provide the diverse repertoire of the antigenbinding receptors? Or does the immune system employ a special somatic strategy in lymphocytes, in which the genes are mutated or randomly recombined to generate additional diversity in the repertoire of HL antibody-combining sites?

Fortunately, the initial scientific battle resolved itself by the late 1970's through the molecular genetic work of immunologist professor Susumu Tonegawa, who was awarded the Nobel Prize in 1987. Tonegawa's work turned out to be a mixture of diversity in the germline genes and diversity in the random somatic processes (recombination and mutation). Indeed, it turned out that the essential process for the generation of the enormous diversity of antibodies and $\mathrm{T}$ cell receptors had to be the random somatic process. That is, during our lifetime our bodies 'learn' to fight numerous invaders and generate many new antibody-coding sequences within lymphocytes. Now we know that gene sequences from lymphocytes may be incorporated into germ cells and passed on to the next generation.

To make a long story short, we now present the essential elements of the antibody repertoire problem and the molecular details of the answer. Each antibody heavy chain $(\mathrm{H})$ is a protein constructed of 400 amino acids ( 100 for the $\mathrm{V}$ region and 300 for the $\mathrm{C}$ region) and each light chain (L) consists of about 200 amino acids (100 for the $\mathrm{V}$ region and 100 for the $\mathrm{C}$ region). Since each amino acid is specified on translation by a codon of three bases, this amounts to at least $1800(600 \times 3)$ bases of DNA sequence information necessary to encode each antibody $\mathrm{HL}$ heterodimer, if each $\mathrm{H}$ and $\mathrm{L}$ chain is encoded by a conventional gene. Thus, if there are one million possible different antibodies, a reasonable estimate, based upon our current knowledge - this would mean that the human genome will need to devote almost 2 billion $\left(1800 \times 1.000 .000\right.$ or $\left.1.8 \times 10^{9}\right)$ bases of DNA sequence information dedicated just to encoding the possible repertoire of antibodies. If we just restrict the calculation to the essential encoded information, that is to the critical $\mathrm{V}$ regions which comprise the antigen-binding site of the molecule, this number would be reduced to about $0.6 \times 10^{9}$, which is about $1 / 3$ of the whole human genome, which has a maximum of 3 billion bases. In other calculations one would end up with between $1 / 6$ and $1 / 2$ of the DNA sequence space devoted to encoding antibody molecules, and that would be a logical impossibility. 


\section{THE DARWINIAN REVOLUTION}

The Darwinian revolution has been a resounding success in biology since its inception. The problem with great scientific revolutions is that they frequently become unmovable dogma. For a while the dogma maybe useful to clarify some points, but soon an 'establishment' form, the individual scientists find it impossible to break ranks, for their careers and financial livelihood are put at great risk. This is opposite to Galileo's legacy, and yet it is an accurate statement on the human condition.

Today it is an unfortunate fact that Neo-Darwinian ideas have evolved into almost a religion in certain quarters, particularly among population geneticists. The New Lamarckian soma-germline-gene-feedback loops (Steele, 1979 especially) and the extra-nucleic L system inheritance are passionately resisted. Some embryologists and population geneticists are still wedded to the neutral theory of molecular evolution of Kimura (1983). Is it true that the presence of degenerate third-position 'silent' changes within a codon being considered as evidence for random genetic drift of mutant forms of a gene, which are selectively neutral, holds water? The very high rate of 'silent' base changes in mammalian housekeeping genes (e.g., histone genes) certainly indicates powerful natural selection conserving the function of the protein.

Why discard Steele's "Somatic Selection and Adaptive Evolution: On the Inheritance of Acquired Characters" (1979) or the soma-to-germline concept with its undoubtedly great explanatory power for the evolution and structure of the $\mathrm{V}$ region gene families of the vertebrate immune system?

The events that are required for the soma-to-germline theory are: 1) The appearance of the mutation in somatic DNA/RNA, 2) Its cellular selection by antigens, 3) Its transport to the germ cells, 4) The copying of RNA into DNA (the copied product is called cDNA if it comes from reverse transcription, and 5) The physical, genetic recombination event leading to the integration of the mutated somatic cDNA copy into germline DNA.

Actually for a long time it has been accepted that contact between B lymphocytes and foreign antigen produces large quantities of endogenous harmless retroviruses carrying the enzyme reverse transcriptase. What the Somatic Selection theory holds is that the immune response system could be the scenario where the acquired somatically mutated V sequences in the lymphocytes' nucleus go back into the germline, thus enriching genetic variability for future generations; the endogenous retrovirus is the sophisticated vector mediating genetic communication between soma and sex cells.

In the Proceedings of the National Academy of Sciences, USA, S. Bartl, David Baltimore and Irving Weissman (1994) speculated that genes could be transmitted between species by viral infection. Barbara McClintock (1978) had informally maintained the same position many times. Implicit in these speculations is the penetration of Weissman's barrier and through it the possible contribution of the vertebrate immune system.

\section{REFERENCES}

Bartl, S., Baltimore, D. and Weismann, I.L. (1994). Molecular evolution of the vertebrate immune system. Proc. Nat. Acad. Sci. USA 91: 10769-10770.

Blackstone, N.W. and Ellison, A.M. (1998). Metazoan development and levels of selection. Unpublished manuscript (cited by Richard E. Michod, Darwinian Dynamics, 1999, Princeton University Press, Princeton, NJ, USA).

Buss, L.W. (1987). The Evolution of Individuality. Princeton University Press, Princeton, NJ, USA.

Campbell, R.D. (1983). Identifying Hydra species. In: Hydra: Research Methods (Lenhoff, H.M., ed.). Plenum Publishing Corporation, New York, NY, USA, pp. 19-28.

Cullis, C.A. (1984). Environmentally induced DNA changes. In: Evolutionary Theory: Paths into the Future (Pollard, J.W., ed.). John Wiley, London, England, pp. 203-216. 
Davidson, E.H., Peterson, K.J. and Cameron, R.A. (1995). Origin of bilateral body plans: evolution of developmental mechanisms. Science 270: 1319-1325.

Eigen, M. and Schuster, P. (1979). The Hypercycle, a Principle of Natural Self-Organization. Springer-Verlag, Berlin. Ewer, R.F. (1948). A review of the Hydridae and two new species from Natal. Proc. Zool. Soc. Lond. 118: 226-244.

Godelle, B. and Reboud, X. (1995). Why are organelles uniparentally inherited? Proc. R. Soc. Lond. B. Biol. Sci. 259: 27-33.

Grayson, R.F. (1971). The freshwater Hydra of Europe. I. A review of the European species. Arch. Hydrobiol. 68: 436-449.

Hoekstra, R.F. (1990). Evolution of uniparental inheritance of cytoplasmic DNA. In: Organizational Constraints on the Dynamics of Evolution. (Maynard Smith, J. and Vida, G., eds.). Manchester University Press, New York, NY, USA, pp. 269-278.

Hoenigsberg, H.F. (1992). Epi- \& extranucleic inheritance. Lamarckian inheritance. Evol. Biol. 6: 225-233.

Hoenigsberg, H.F. and Parkash, R. (1995). Comparative genetic structure and spatial patterns in the evolution of cosmopolitan and local species. Evol. Biol. 8: 231-242 and 9: 215-236.

Kimura, M. (1983). The Neutral Theory of Molecular Evolution. Cambridge University Press, Cambridge, England.

Landman, O.E. (1991). The inheritance of acquired characteristic. Annu. Rev. Genet. 25: 1-20.

Maynard Smith, J. and Szathmáry, E. (1995). The Major Transitions in Evolution. W.H. Freeman, San Francisco, CA, USA.

McClintock, B. (1978). Mechanisms that rapidly reorganize the genome. Stadler Symp. 10: 25-48.

Michod, R.E. (1982). The theory of kin selection. Annu. Rev. Ecol. Syst. 13: 23-55.

Michod, R.E. (1997a). Cooperation and conflict in the evolution of individuality. I. Multi-level selection of the organism. Am. Nat. 149: 607-645.

Michod, R.E. (1997b). Evolution of the individual. Am. Nat. 150: S5-S21.

Michod, R.E. (1999). Darwinian Dynamics. Princeton University Press, Princeton, NJ, USA.

Morrell, V. (1996). Genes versus teams. Science 273: 739-740.

Pollard, J.W. (1984). Is Weismann's Barrier Absolute? In: Beyond Neo-Darwinism: Introduction to the New Evolutionary Paradigm (Ho, M.W. and Saunders, P.T., eds.). Academic Press, London, England, pp. 291-315.

Prasad, N. and Mookerjee, S. (1986). Morphogenetic analysis of ecotypes of Indian Hydra. Part III. Understanding Physiological Responses. Zoological Survey of India. Tech. Monog. No. 13: 75-108.

Pren Das, O., Levi-Minzi, S., Koury, M., Benner, M. and Messing, J. (1990). A somatic gene rearrangement contributing to genetic diversity in maize. Proc. Nat. Acad. Sci. USA 87: 7809-7813.

Ransick, S., Cameron, R.A. and Davidson, E.H. (1996). Postembryonic segregation of the germ line in sea urchins in relation to indirect development. Proc. Nat. Acad. Sci. USA 93: 6759-6763.

Rastogy, N. and Pandey, M. (1992). Statistical analysis of geographical variability in 16 ecotypes of Indian Hydra. Evol. Biol. 6: 195-204.

Rothenfluh, H.S. (1995). A memory lymphocyte-specific soma-to-germline genetic feedback loop. Immunol. Cell Biol. 73: 174-180.

Rothenfluh, H.S. and Steele, E.J. (1993). Origin and maintenance of germ-line V-genes. Immunol. Cell Biol. 71: $227-232$.

Rothenfluh, H.S. and Steele, T. (1993). Lamarck, Darwin and the immune system. Today's Life Sci. 5: 8-15 and 5: 16-22.

Rothenfluh, H.S., Blanden, R.V. and Steele, E.J. (1995). Evolution of V genes: DNA sequence structure of functional germ-line and pseudogenes. Immunogenetics 42: 159-171.

Sapp, J. (1987). Beyond the Genetic Cytoplasmic Inheritance and the Struggle for Authority in Genetics. Oxford University Press, New York, NY, USA.

Steele, E.J. (1979). Somatic Selection and Adaptive Evolution: On the Inheritance of Acquired Characters. Williams and Wallace, Toronto. 2nd edn. University of Chicago Press, Chicago, IL, USA.

Steele, E.J. and Cairns, J. (1989). Dispute resolved. Nature 340: 336

Steele, E., Blanden, R. and Rothenfluh, H. (1996). How have antibody genes evolved? Aust. Sci. 17: 46-49.

Steele, E.J., Lindley, R.A. and Blanden, R.V. (1998). Lamarck's Signature, How Retrogenes are Changing Darwin's Natural Selection Paradigm. Helix Books, Perseus Books, Reading, MA, USA.

Temin, H.M. (1989). Retrons in Bacteria. Nature 339: 254-255.

Williams, G.C. (1996). Adaptation and Natural Selection. New Edition. Princeton University Press, Princeton, NJ, USA.

Wood Jones, F. (1943). Habit and Heritage. Kegan Paul, Trench, Trubner \& Co., London, England.

Xiao, S., Zhang., Y. and Knoll, A.H. (1998). Three dimensional preservation of algae and animal embryos in a Neoproterozoicphosporite. Nature 391: 553-559.

Zoragi, G. and Spadafora, C. (1997). Integration of foreign DNA sequences into mouse sperm genome. DNA Cell Biol. 16: 291-300. 